1 Title: Mosaic evolution, preadaptation, and the evolution of evolvability in apes

2 Author: Caroline Parins-Fukuchi*1

$3{ }^{1}$ Department of Geophysical Sciences, University of Chicago, Chicago, IL, USA

4 *Author for correspondence: parinsfukuchi@uchicago.edu

5 Data archived online: https://figshare.com/articles/parins-fukuchi_supp_zip/8063726

6

\title{
7 Acknowledgements
}

8 I wish to thank D Alvarado-Serrano, M Cosman, CW Dick, DC Fisher, M Foote, E

9 Greiner, D Jablonski, LM MacLatchy, H Marx, J Saulsbury, SA Smith, G Slater, N

10 Walker-Hale, M Webster for conversations that have greatly benefitted this work. I also

11 thank S Worthington for help accessing his skeletal measurements that comprised a large

12 portion of the character matrix analyzed here. The manuscript benefitted immensely from

13 careful reviews provided by $\mathrm{P}$ Wagner and PD Polly.

14

15

16

17

18

19

20

21

22 
23 Abstract: A major goal in post-synthesis evolutionary biology has been to better

24 understand how complex interactions between traits drive movement along and facilitate

25 the formation of distinct evolutionary pathways. I present analyses of a character matrix

26 sampled across the haplorrhine skeleton that revealed several modules of characters

27 displaying distinct patterns in macroevolutionary disparity. Comparison of these patterns

28 to those in neurological development showed that early ape evolution was characterized

29 by an intense regime of evolutionary and developmental flexibility. Shifting and reduced

30 constraint in apes was met with episodic bursts in phenotypic innovation that built a wide

31 array of functional diversity over a foundation of shared developmental and anatomical

32 structure. Shifts in modularity drove dramatic evolutionary changes across the ape body

33 plan in two distinct ways: 1) an episode of relaxed integration early in hominoid

34 evolution coincided with bursts in evolutionary rate across multiple character suites; 2)

35 the formation of two new trait modules along the branch leading to chimps and humans

36 preceded rapid and dramatic evolutionary shifts in the carpus and pelvis. Changes to the

37 structure of evolutionary mosaicism may correspond to enhanced evolvability that has a

38 'preadaptive' effect by catalyzing later episodes of dramatic morphological remodeling.

39 Keywords: integration, evolvability, preadaptation, modularity, hominoidea

\section{Introduction}


46 by the availability of unoccupied 'adaptive zones', classically represented as peaks on a

47 fitness landscape (Wright 1932, Simpson 1944). Frequently referred to as 'ecological

48 opportunity' (Losos 2010), the opening of new adaptive zones through shifts in biotic

49 interactions and the environment is often invoked to explain large evolutionary radiations

50 (Rainey and Travisano 1998, Yoder et al., 2010, Wagner and Harmon et al 2012, but see

51 Slater 2015). The role of ecological processes in generating morphological novelty can be

52 contrasted with an increasing focus on 'constructional' factors that include functional

53 interactions and architectural constraints (Seilacher 1970, 1991). While character

54 evolution may sometimes reflect simple optimization processes when natural selection

55 operates independently on separate traits, the evolutionary pathways realized in nature are

56 also constrained by the complex interactions between functionally dependent traits and

57 the limitations of an organism's developmental architecture. When placed into a broader

58 evolutionary literature, these limiting factors might be coarsely partitioned into

59 developmental (Gould and Lewontin 1979, Alberch 1980, Olson 2012) and functional

60 (Charlesworth et al., 1982, Maynard-Smith et al., 1985, Arnold 1992) constraints.

Developmental and functional constraints limit the capacity for a clade to evolve

62 novel phenotypes. Together, these factors might be said to determine the level of

63 'constructional opportunity' available at a given time. Contrasted to ecological

64 opportunity, constructional opportunity reflects how the structure of developmental and

65 functional interactions between characters limits the 'evolvability' of a clade-its

66 capacity to evolve novel phenotypes. While ecological opportunity is often evoked as a 
67 limiting factor on a clade's phenotypic diversification, constructional opportunity, as

68 measured by the overall level of constraint and internal integration between phenotypes,

69 may also limit the range of evolutionary modifications available to a population faced

70 with ecological pressure.

71 Evolutionary constraints are shaped by shared developmental pathways,

72 multivariate selection, and underlying genetic processes such as epistasis and pleiotropy.

73 Such interactions between traits and genes often create a common tendency to drive the

74 formation of modules of characters that are internally integrated in their evolutionary

75 trajectories. Such integration is typically identified by calculating numerical correlations

76 in their variance (Olson and Miller 1958). Integration between traits constrains the

77 combination of phenotypes accessible to each. For example, for two traits that are

78 strongly positively integrated, it is unlikely to observe an individual that possesses

79 phenotypes for each trait that lie at opposing ends of the spectrum of variation. As long as

80 this correlation exists, the evolution of the organism is constrained in the sense that only

81 certain combinations of phenotypes are accessible. At the population level, integration

82 can be caused by multivariate selection or physical/constructional linkages such as

83 pleiotropy or allometry (Cheverud 1984). However, it is generally not possible to

84 distinguish between these in macroevolutionary study systems.

85 Phenotypic integration generally occurs in a modular fashion (Wagner et al.

86 2007). Instead of covarying uniformly across the entire organism, integration often

87 manifests as a patchwork of modules of internally integrated traits that are free to evolve

88 largely independently (Goswami et al. 2014, 2015). Such modular structures lead to the 
89 pattern of mosaic evolution when separate modules evolve at different rates as lineages

90 diverge (Felice and Goswami 2017, Parins-Fukuchi, in press). When analyzed

91 phylogenetically, these patterns result in 'mosaic disparity' - a scenario where individual

92 modules of traits each display a unique pattern in phylogenetic disparity across lineages

93 (Fig. 1).

94 The related concepts of mosaicism, constraint, and integration can help to explain

95 the emergence of phenotypic novelty across clades. For example, when ecological

96 opportunity is abundant, evolutionary radiations can be driven primarily by the release of

97 constraints (Wagner et al. 2003). In addition, bursts in evolutionary rate can also coincide

98 with the break-up of existing modules and the subsequent formation of a new modular

99 structure (Wagner 2018). Characterizing the role of integration and mosaic evolution in

100 shaping diversity across clades will ultimately contribute to a deeper understanding of

101 phenotypic evolution at the constructional level. This may improve our understanding of

102 evolutionary processes more generally by revealing the extent to which fluctuations in

103 constructional opportunity contribute to macroevolutionary patterns alongside ecological

104 opportunity.

105 In this study, I sought to determine the extent to which the evolutionary

106 trajectories blazed by ape species were shaped by early shifts in the structure of

107 integration and the timing of development. To address these questions, I reconstructed the

108 mosaic macroevolutionary patterns in morphological, neurological, and developmental

109 phenotypes across living and fossil great apes throughout the Miocene. To help place

110 these empirical results in a theoretical context, I also present a set of simple simulations 
111 to generate a theoretical expectation for the patterns in disparity expected when

112 Markovian diffusion is constrained by trait integration imposed by both functional and

113 structural/developmental interactions. Taken together, these analyses suggest the

114 capability for shifting patterns in constraint imposed by integration to create

115 constructional opportunities that generate repeated and dramatic episodes in

116 morphological innovation.

117 Apes have been extensively studied in the context of both developmental

118 constraint and environmental adaptation. Young et al. (2010), found that apes display

119 weak integration between the fore- and hindlimb in comparison to cercopithecoids.

120 Reduced integration can confer greater functional flexibility, and so may have facilitated

121 the proliferation of the diverse locomotor function across living and fossil apes. Changes

122 in developmental timing have also been important in ape evolution. Developmental

123 heterochrony appears to have driven the emergence of many key aspects of hominin

124 morphology in the cranium and post-cranium (Gould 1977, Berge 1998). This long-

125 standing interest in the developmental basis of ape evolution has not excluded

126 environmental hypotheses. Environmental fluctuations throughout the Miocene and

127 Pliocene have also frequently formed the basis for adaptive hypotheses across apes

128 (Andrews 1992, Ruff 1994, Michel et al., 2014). The body plans of hominins, and

129 undoubtedly apes in general, have been shaped through modifications to separate suites

130 of traits occurring at different times (Holloway 1973, McHenry 1975). These diverse

131 threads suggest that a multiplicity of causes, perhaps including both ecological

132 opportunity and constructional factors, has shaped the evolution of hominoid body plans. 
133 As a result, apes are an excellent exemplar taxon in which to pluralistically examine the

134 mosaic patterns in constraint and innovation that shape body plans in living and fossil

135 vertebrates.

136 Methods

137 Morphological data

138 I gathered a dataset of 149 quantitative morphological traits from the literature

139 (Lewton 2010, Worthington 2012) spanning the cranium, forelimb, and pelvis. These

140 traits were sampled across 10 extant taxa. I also collected fossil cranial and forelimb traits

141 from Worthington (2012), retaining all taxa with at least 25\% matrix occupancy. Taxa

142 were all studied at the generic level, with each extant genus represented by a single

143 exemplar species. Each morphological trait represented either a dimensionless ratio or

144 geometric mean, depending on how the author of each dataset handled intraspecific

145 variation and body mass correction. I scaled the traits to display an empirical variance of

1461 across all taxa. This simplified the analyses by reducing the complexity of the dataset,

147 while retaining the same comparative information. Since the original traits were

148 dimensionless, both the unscaled and the scaled datasets would facilitate examination of

149 relative, rather than absolute, evolutionary rates, but the scaled traits improve Markov-

150 chain Monte Carlo (MCMC) mixing and simplify the identification of mosaic suites.

\section{Neurological data}

152 To directly study the evolutionary patterns in related developmental and

153 phenotypic traits, I also gathered neurological data from the literature (Capellini et al.,

154 2010, Boddy et al., 2012). For neurological phenotypes, I collected encephalization 
155 quotients (EQ) estimated in 76 primate species by Boddy et al. (2012). EQ is calculated

156 by fitting a nonlinear model to brain size and body size and taking the deviations

157 (residuals) from the best fit curve and so measures the enlargement of brain size while

158 controlling for allometry with body size. I compared EQ patterns to those in a postpartum

159 encephalization development (ED) metric calculated as:

$160 E D=E Q_{\text {adult }}-E Q_{\text {neonatal }}$

161 ED is therefore intended to reflect the amount of brain tissue grown by members of each

162 species following birth relative to their body size. The ED metric was scaled to unit

163 variance across all sampled taxa. Neonatal brain and body mass data representing 24

164 primate species were acquired from Capellini et al. (2010).

165 Fossil placement and divergence time estimation

166 Since the evolutionary relationships between the extant taxa represented in the

167 morphological dataset have all been extensively studied and are well-resolved, I used

168 these as a fixed scaffolding to place the fossil taxa. Fossil placements were inferred from

169 the continuous trait dataset using the cophymaru program (Parins-Fukuchi 2018). I used

170 the 'binary weights' procedure to filter out reliable traits from ones likely to mislead by

171 using the extant tree as a fixed point of reference. Markov-chain Monte Carlo (MCMC)

172 simulations were run for 1,000,000 generations and checked manually for convergence.

173 The posterior tree sample was summarized as the maximum clade credibility tree using

174 sumtrees.py (Sukumaran and Holder 2010).

175 For downstream comparative analyses, I also estimated divergence times on the

176 full 19-taxon tree. I downloaded cytochrome B sequences from Genbank representing 
177 each of the 10 extant taxa. Molecular dating was performed using Beast version 2.4.8

178 (Bouckaert et al. 2014) using the fossilized birth-death (FBD) prior. The topology was

179 fixed to the summary tree generated during the fossil placement step. Temporal

180 occurrence ranges for the fossil taxa were assembled from the literature. These fossil and

181 extant occurrence ranges were used in the dating analysis to infer the diversification and

182 sampling rate parameters used in the FBD prior.

183 Measuring integration throughout the hominoid radiation

184 As an evaluation of the overall strength of integration in the skeletal traits across

185 the haplorrhine phylogeny, I estimated ancestral states for each character under Brownian

186 motion along a dated tree of nine extant haplorrhine taxa (Pan paniscus was removed

187 because it was missing pelvic measurements). I then estimated the strength with which

188 the reconstructed phenotypes at each internal node were correlated with the values at

189 each descendant node or tip. The correlations indicated how strongly the reconstructed

190 evolutionary changes in skeletal traits were integrated along each branch in the tree.

191 Stronger correlations corresponded to tighter overall integration. The Python script that I

192 developed for the reconstruction of ancestral states and calculation of character

193 correlations is available in the data supplement.

194 Evolutionary rates across mosaic morphological suites

195 I used the greedo program to recover mosaic patterns in disparity from the

196 continuous trait dataset (Parins-Fukuchi 2019). Greedo is a phylogenetic

197 clustering/mixture model estimation approach that uses the Akaike information criterion

198 (AIC) to iteratively merge and split clusters of traits to find a best-supported set of 
199 character suites based on shared patterns in disparity (i.e., where integrated traits within

200 the same module are similarly disparate across taxa), where each is represented by a tree

201 with branch lengths (Fig. 1). Traits that display the highest improvement in log-likelihood

202 when assigned to a separate cluster are prioritized during the splitting steps to first

203 identify likely sources of heterogeneity in the dataset. During the merging step, only

204 clusters that show an improved AIC score when placed under the same model are joined.

205 This process recovers an estimate of the number of suites, the membership of each trait,

206 and a tree with branch lengths scaled to units of disparity for each suite. Fossil taxa were

207 not included in the mosaic analyses, because they were too fragmentary in their sampling

208 to inform disparity patterns when the traits were split into suites. I performed many runs

209 of the greedo procedure to avoid presenting results obtained from a suboptimal peak on

210 the likelihood surface. Since several of the top clusterings yielded close AIC support, I

211 performed an additional model-averaging step using Akaike weights to summarize the

212 results as a graph where each trait occupies a node where each link is weighted to reflect

213 the total weighted AIC support across all the models. Comparative analyses were

214 performed using a single summary clustering that resulted from application of the

215 Markov clustering (MCL) algorithm to the support network (Dongen 2000).

216 To examine the anatomical composition of the mosaic suites of characters, I

217 performed a statistical enrichment procedure to identify skeletal regions that were over

218 and under-represented in each suite relative to the proportions for each present in the

219 entire dataset. The proportion of the 149-trait dataset represented by each skeletal region

220 was used to generate a set of 'expected' values. I then compared these to the observed 
221 proportions within each suite to examine trends in the deviations of the proportions

222 occupied by each region. I did not assess the statistical significance of these deviations,

223 because 1) my aim was to examine the general patterns in the composition of each

224 character suite rather than to determine absolute significance, and 2) the small sample

225 size of the dataset generated expected chi-square test statistic values that were nearly

226 entirely $<5$, making the test inappropriate. To examine the relative contributions of

227 separate anatomical regions in shaping the macroevolutionary patterns reconstructed in

228 each suite, I also performed a principal component analysis (PCA) on the traits contained

229 within each suite. I then transformed the loadings to calculate the total variance

230 contributed by each trait across all axes of the PCA, summing them to produce the

231 contribution of each anatomical region.

232 I transformed the branch lengths for each mosaic suite, which were scaled to

233 reflect disparity (total variance, $v$, accumulated over time $t$ ), to rates ( $v$ per unit $t$ ) using

234 the results from the divergence time analysis. I also calculated evolutionary rates

235 averaged over the entire morphological dataset while including the fossil taxa to coarsely

236 reconstruct evolutionary tempo throughout the Miocene. Although this step missed

237 valuable information recovered by the mosaic analyses, including the fossil taxa in this

238 manner enabled finer resolution into the coarse patterns in evolutionary tempo throughout

239 the Miocene.

240 Evolutionary rates in neurological data

241 I estimated macroevolutionary rates across primates in ED and EQ using BAMM

242 version 2.5.0 (Rabosky 2014). Since the trees that I constructed for the morphological 
243 analysis contained only a fraction of the taxa present in the neurological datasets, I used

244 the dated primate supertree packaged in BAMM that was originally sourced from Mos

245 and Mooers (2006) and pruned the tips to match the taxa present in each dataset. MCMC

246 simulations were run until the estimated sample size (ESS) well exceeded 200 for each

247 parameter. Results were presented by plotting the mean rate estimated along each branch

248 and the maximum a posteriori configuration of rate shifts using BAMMtools version

249 2.5.0 in R (Rabosky et al., 2014).

250 Lineage diversification rate analyses

251 I performed analyses of origination and extinction rates in hominoid and

252 cercopithecoid fossil records to examine the correspondence between lineage

253 diversification and the patterns in disparification emphasized in the analyses of

254 morphological rates and mosaicism. Fossil occurrence data spanning primates were

255 acquired from the Paleobiology Database (paleobiodb.org) on July 31, 2019. This dataset

256 was partitioned into two subsets: one encompassing Hominoidea and the other

257 representing Cercopithecoidea. Rates of origination and extinction were inferred from

258 each subset separately using PyRate (Silvestero et al. 2014). The inferential model was

259 constrained to contain uniform origination and extinction rates across time, thus ignoring

260 the possibility of diversification shifts within each clade. Preservation was constrained to

261 being time-homogeneous and was assumed to be uniform within each clade. While

262 ignoring potential information regarding diversification rate heterogeneity through time,

263 this simplified model matches classic paleobiological work that assumes simple birth-

264 death-sampling models and was therefore appropriate for the comparison of average 
265 diversification rates in sister clades while avoiding possible model overfitting that may

266 obfuscate the straightforward test sought here.

267 Simulated Markovian diffusion

268 To examine the expected effect of constructional opportunity stemming from both

269 developmental and functional constraint on phenotypic evolution, I designed a set of

270 evolutionary simulations based on a simple Markovian diffusion. In this system,

271 quantitative traits belonging to a single population of organisms evolve stochastically

272 along a fitness landscape. Each generation, values for each trait are proposed. Values that

273 increase the overall fitness of the population are accepted. To mimic the effect of drift,

274 values that decrease fitness are also accepted with some (user-specified) probability.

275 Constraint stemming from developmental integration caused by pleiotropy can be

276 mimicked by proposing values for traits that covary, while functional constraints are

277 modeled by evolving traits along a shared, multivariate adaptive landscape, modelled

278 here using a mixture of multivariate Gaussian distributions. In a completely

279 unconstrained system, each trait evolves according to its own univariate landscape, with

280 its value drawn independently of all others in each generation. Performing simulations in

281 this way facilitated an illustration of the potential for functional and developmental

282 integration to constrain evolutionary rates in an adaptive landscape. Although

283 distinguishing between these modes of integration is generally difficult or impossible in

284 paleontological study systems, the simulations performed here provide insight by testing

285 1) the potential for varying systems of integration to catalyze or constrain adaptive

286 change and 2) the extent to which functional and developmental integration generate 
287 overlapping and distinct patterns in evolutionary rate and disparity. When placed in a

288 macroevolutionary context, the simulations here should be thought of as a demonstration

289 of the possible microevolutionary processes that may have driven the higher-level

290 patterns in evolutionary rate occurring along a single phylogenetic branch in the mosaic

291 analyses. The script used to generate these simulations is available in the data

292 supplement.

\section{Results and Discussion}

294 Strength of integration throughout the haplorrhine radiation

295 Calculating character correlations from the skeletal data along each branch of the

296 extant haplorrhine phylogeny revealed a single episode of reduced integration at the root

297 of Hominoidea, where the correlation decreased from 0.12 at the ancestor of catarrhines,

298 to -0.04 at the root of hominoids (Fig. 2). This suggests that the reduction in integration

299 across apes between the fore- and hindlimbs revealed by Young and colleagues (2009)

300 extended across the skeleton along the branch leading to the stem hominoids. Such

301 episodic relaxed integration was not detected in the other haplorrine lineages, with

302 cercopithecoids (0.2-0.33 correlation) and platyrrhines ( $0.16-0.31$ correlation) displaying

303 higher integration than hominoids (-0.04-0.25 correlation) overall. However, it is possible

304 that more complete sampling of taxa and characters across both of these clades may

305 reveal changes in modularity and integration not detected here.

306 Composition of mosaic suites

307 The analysis of mosaic disparity recovered five suites of traits (Fig 3b). The

308 cranium was the largest contributor of both traits and variance across all suites except for 
$309 \mathrm{C} 4$, which was represented by the pelvis alone (Fig. 3b and 3c). This reflects the higher

310 sampling of cranial traits in the dataset. While the cranium has previously been shown to

311 display substantial modularity (Felice and Goswami 2017), the results here suggest that

312 individual cranial modules may result from the formation of broader evolutionary

313 complexes shared with the post-cranium. The suites were distinct in their composition

314 across postcranial anatomy: C0 was represented strongly by the wrist, ulna, and humerus;

315 C1 was represented postcranially by the scapula; C2 by carpal and pelvic traits. The

316 cranium was represented even more strongly than expected in C3 and contributed nearly

317 half of the variance (Fig. 3b).

318 Rates of morphological evolution

319 The phylogenetic rate calculations for each suite revealed a strong pattern of

320 evolutionary mosaicism (Fig. 3a). Three of the suites, C0, C1, and C3, displayed the

321 highest rates after the divergence of the great apes from hylobatids, but before the

322 divergence of gorillas from humans and chimpanzees. The two suites that did not

323 experience the shared great ape rate increase, $\mathrm{C} 2$ and $\mathrm{C} 4$, experienced large bursts in

324 Homo. This finding is consistent with general knowledge of human evolution, as both

325 suites are dominated by cranial traits and pelvic traits related to birthing and locomotor

326 function (Table S1).

327 The rate analyses (Fig. 3a) show that humans have experienced major

328 macroevolutionary bursts. However, the unique characteristics of the human body plan

329 are built over an anatomical structure shared by all great apes that was shaped in the

330 Miocene during similarly dramatic episodes. For example, although suite C0 increased in 
331 evolutionary rate along the human branch, its evolution was shaped by a sustained period

332 of elevated rate between 20 and 10 million years ago. This suggests that much of the

333 'groundwork' underlying the derivation of humans' unique features may predate the

334 divergence between our lineage and chimpanzees'. The mosaic analysis also

335 demonstrates that substantial visible phenotypic novelty can result from the evolutionary

336 remodeling of a small subset of the anatomy. The most elevated evolutionary rates in

337 humans were observed in suites $\mathrm{C} 2, \mathrm{C} 3$, and $\mathrm{C} 4$, which cumulatively comprise fewer than

338 one-third of the traits sampled in the matrix (Table S1). C4, the smallest suite (10 traits)

339 displayed by far the largest increase in rate along the Homo lineage. Perhaps notably, C4

340 also was the most static character suite throughout the earlier stages in the hominoid

341 radiation. The mosaic pattern recovered here shows that the uniqueness of the human

342 body plan was shaped by dramatic evolutionary shifts in only a small number of traits.

343 While the large amount of missing data among the fossil taxa made an additional

344 mosaic analysis infeasible, their inclusion revealed an otherwise hidden shift in

345 evolutionary rate that occurred at the root of Hominoidea (approximately 30 million years

346 ago). The fossil data also recapitulated the burst in evolutionary rate during the mid-

347 Miocene that was displayed by C0 and C1 in the mosaic analysis (Fig. 3 and 4). When

348 averaging over all the traits and including fossil taxa, these two episodes are the most

349 dramatic macroevolutionary events when tracing the evolutionary lineage leading to

350 Homo, suggesting the importance of early shifts in the ape body plan in shaping the

351 functional morphologies of living taxa. 
353 corresponded to increased evolvability conferred by relaxed integration among early

354 apes. However, comparison of the human-specific evolutionary rates in cranial, pelvic,

355 and neurological traits to the phylogenetic character correlations in Figure 2 shows that

356 the elevated morphological rates in humans were immediately preceded by an increase in

357 the strength of integration. This illustrates an alternative means through which shifts in

358 systems of modularity promote rapid and dramatic evolutionary remodeling of

359 morphology. The suites that showed the most dramatic rate increases in humans, C2 and

$360 \mathrm{C} 4$, were evolutionarily inactive prior to the most recent common ancestor (MRCA) of

361 chimps and humans. This suggests that, while early ape evolution was characterized by

362 relaxed integration leading to rapid evolutionary change in existing modules, human

363 morphological evolution was shaped by the formation of two small modules of characters

364 from the pelvis and carpus that underwent an initial rate increase following their

365 formation along the branch leading to chimps and humans and then a more dramatic rate

366 increase in hominins. Although the overall structure of integration was well-defined

367 along the chimp-human MRCA branch, the formation of new, smaller modules

368 contributed to dramatic changes across the body plan in humans. The occurrence of these

369 initial shifts in modularity and rate along the chimp-human MRCA shows that

370 chimpanzees share the modified structure of integration that facilitated the evolution of

371 key human traits. This result is surprising given the typical assumption that chimpanzees

372 are morphologically more similar to other great apes than are humans.

373 Rates of neurological evolution 
375 Homo displays the highest rate of both EQ and ED evolution. However, the human rate

376 shift in both traits occurred as part of an older trend of rapid neurological evolution in

377 African apes. For EQ, humans experienced a substantial increase in evolutionary rate

378 relative to the rest of the African apes, despite the statistical evidence for a shared rate

379 shift at the root of the clade (Fig. 5b). The shared macroevolutionary regime shared by all

380 African apes is most apparent in encephalization development (Fig. 5a), where the

381 estimated evolutionary rate in Homo increased only slightly after splitting from Pan (Fig.

382 5c). It appears that the increased encephalization developed throughout the post-natal

383 period in humans reflects a general trend among great apes in the evolutionary plasticity

384 of neurological development. As a result, the ability to develop a relatively large mass of

385 brain tissue does not itself appear to be a strong limiting factor in the evolution of large

386 encephalization in humans. Instead, the morphological analyses suggest that pelvic traits

387 (C4 in Fig. 1) demanded a more dramatic alteration in macroevolutionary regime,

388 requiring both the formation of a new suite of characters and massive burst in rate.

389 Structural and ecological opportunity in ape evolution

390 The elevated evolutionary rates experienced by apes early in their divergence

391 correspond to a general relaxation of constraint in early Miocene stem and ancestral

392 hominoids. The reduction in integration across the skeleton and burst in the evolutionary

393 rate of neurological development that occur at the base of the hominoid clade correspond

394 in timing to the elevated rates in morphological evolution observed in most of the skeletal

395 characters examined here (Fig. 3 and 4). Freed from previous functional and 
396 developmental limitations, ape body plans would have rapidly diversified when placed in

397 the context of the repeated environmental fluctuations that occurred throughout the mid

398 and late Miocene (Michel et al. 2014, Hunt 2016) before becoming functionally canalized

399 through development and stabilizing selection. This pattern is consistent with a scenario

400 in which morphological features evolve by stochastic diffusion on a Simpsonian adaptive

401 landscape that is alternately dampened and released by relaxed and shifting patterns in

402 character integration.

403

Rapid and frequent environmental fluctuations throughout the Miocene suggest a

404 possible abundance of available adaptive zones during the evolution of both

405 cercopithecoids and hominoids. Paleoecological information extracted from deposits

406 containing key hominoid taxa are so variable that environmental turnover often outpaces

407 the effects of time-averaging (Michel et al. 2014). Early Miocene ape and monkey

408 species evolved in overlapping environmental conditions and shared similar

409 ecomorphological and dietary habits. However, as their lineages diverged, apes evolved a

410 diverse set of locomotor suites, while evolution in locomotor features has remained

411 comparatively static across old world monkeys (Hunt 2016). The patterns in

412 developmental and mosaic morphological evolution revealed here suggest that the

413 substantial differences between the two taxa in phenotypic disparity were shaped more by

414 differences in patterns in integration and constraint rather than from the ecological

415 opportunities available to either. While hominoids and cercopithecoids inhabited a

416 similar range of habitats throughout their evolution, and so likely experienced a similar

417 abundance of ecological opportunity, greater constructional opportunity is a 
418 distinguishing feature that correlates with hominoids' vast disparity in skeletal

419 morphology.

420 The scenario entertained here for apes is consistent with recent work that suggests

421 that the initial burst in phenotypic disparity that often accompanies the origin of a new

422 lineage can result from an early relaxation of constraints through the dissolution of

423 integrated modules that is followed by reformation of character suites that lead to rapid,

424 correlated evolutionary changes between constituent characters (Wagner 2018). In the

425 cited work, the author distinguishes this 'breakup-relinkage' model with one where

426 evolutionary rates are elevated by a relaxation of constraint alone. The 'breakup-

427 relinkage' model is directly supported by the phylogenetic integration pattern, which

428 show that an initial decrease in integration at the root of hominoids was followed by an

429 increase at the following node (Fig. 2). Therefore, the recanalization of functional

430 variation into a set of suites with distinct functional and developmental properties early in

431 hominoid variation is likely equally important to the initial relaxation in constraint in

432 having driven the remarkable morphological disparity observed across hominoid species.

433 "Preadaptation" and developmental enablers in apes

434 Bursts in phenotypic change and diversification are often preceded by

435 evolutionary enablers that facilitate the construction of more diverse morphological forms

436 or fulfill a necessary condition for the later emergence of a more derived trait. Such

437 patterns have been suggested to stem from higher-level processes variously referred to as

438 “preadaptation” (Bock 1959), “exaptation” (Gould and Vrba 1982), and “developmental

439 enablement" (Donoghue 2005). The evolution of developmental, morphological, and 
440 behavioral traits is often hierarchical through functional inter-dependencies and

441 temporally autocorrelated, with changes in traits at relatively lower levels 'setting the

442 stage' for higher-level traits by providing the developmental or structural conditions

443 necessary for their emergence. The stepwise macroevolutionary bursts observed here

444 show that hominoid evolution has been defined by sequential, episodic releases in

445 constraint that likely set the stage for later innovations that compounded upon earlier

446 rearrangements in the structure of developmental and functional integration.

447 The related concepts of preadaptation, exaptation, and developmental enablement

448 all focus on identifying the origin of single characters that facilitate the later emergence

449 of more derived states. However, the patterns presented here suggest that such processes

450 may often be better characterized as reflecting larger changes in developmental and

451 functional character-linkage systems rather than by the sequential derivation of individual

452 characters. The dramatic bursts in evolutionary rate that occur in the largest character

453 suites coincide with or immediately follow the reduction in integration that occurred at

454 the root of hominoids. This suggests that a general shift in functional and/or

455 developmental integration occurred near this time. This shift precedes and, although

456 speculative, may have resulted in a general predisposition across apes that facilitated the

457 later functional divergence of later species into an unusually broad range of habits.

458 Therefore, rather than stemming from a single "preadaptive" character, the morphological

459 disparity of apes and the emergence of features derived in single lineages (such as

460 bipedalism in humans) may be better explained by a shift in developmental and

461 functional integration patterns earlier in ape evolution. 
463 contributed to the emergence of key human traits. The apparent formation of suites $\mathrm{C} 2$

464 and C4, represented disproportionately by the cranium and pelvis, along the branch

465 leading to humans and chimpanzees coincided with an initial burst in evolutionary rate.

466 Both of these events immediately preceded the dramatic bursts in Homo (Fig. 3).

467 Likewise, EQ and ED have been evolving rapidly throughout the ape radiation, with ED

468 experiencing an initial increase in evolutionary rate before the splitting of gorillas,

469 chimps, and humans followed by more dramatic upticks of rate in humans in both traits

470 (Fig. 5c). While distinct in its details, this pattern in chimps and humans provides further

471 evidence for the preadaptive potency of shifts in systems of modularity and integration in

472 driving dramatic anatomical rearrangements. It also illustrates that the broad range of

473 possible evolutionary pathways opened by either an overall reduction in integration (at

474 the hominoid root) or the formation of new modules (in chimps and humans), rather than

475 movement toward a specific set of phenotypes, is the means through which such

476 predispositions facilitate evolutionary remodeling.

477 It is possible that the initial bursts in ED and suite C2 and C3 evolution, coupled

478 with the reduced integration in the MRCA of chimp and humans, may reflect a set of

479 structural rearrangements that facilitated, at least in part, the later emergence of human-

480 specific neurological and cognitive features. This scenario echoes the results of Rice

481 (1998), who demonstrated that humans and chimpanzees, to the exclusion of gorillas,

482 both inherited a shared 'additional' stage of brain development from our most recent

483 common ancestor. It is therefore possible that, in addition to sharing a similar derived 
484 structure of modularity, chimpazees possess more of the neurological and cranial

485 groundwork that contributed to the evolution of humans' particular mode of cognition

486 than has previously been appreciated.

487 The patterns uncovered here suggest that the concepts of preadapation and

488 exaptation may sometimes be more compellingly applied by shifting focus from

489 individual features to patterns in integration and modularity across broad suites of

490 characters. Although the concept is essentially unchanged as applied to integration

491 patterns, it may be more accurate in the example here to refer instead to an 'evolutionary

492 predisposition' given the lack of evidence for a specific sequence of selective regimes

493 (Smith et al. 2018). This semantic argument is likely also valid in more traditional

494 examples of single trait exaptations or preadaptations. And so, a generalization from

495 preadaptation or exaptation to evolutionary predisposition that 1) incorporates integration

496 patterns and 2) shifts emphasis from adaptation, which makes assumptions about the

497 (often unknowable) set of historical microevolutionary processes, to the sequence of

498 observable states in both characters and integration patterns can provide a stronger

499 conceptual basis for future work that considers contingency and sequential order in the

500 evolutionary pathways of complex traits. Although this reconceptualization might

501 superficially appear to de-emphasize evolutionary process in favor of pattern, I would

502 instead argue that constraining the scale of inquiry and incorporating a broader range of

503 biological complexity can facilitate the reconstruction of elusive higher-level

504 macroevolutionary processes that shape organismal diversification and disparification

505 over deep timescales. This formulation also avoids the conceptual and practical 
506 frustrations that occur when attempting to invoke the concepts of preadaptation and

507 exaptation to explain evolutionary history over deep timescales by reducing dependency

508 on knowledge of lower-level population processes.

509 Diversification and disparification in haplorrhine evolution

510 Cercopithecoids have diversified in species number at over twice the rate as

511 hominoids over the same time scale (Table 1). One limitation of these analyses stems

512 from cercopithecoids' somewhat poor fossil record as compared to hominoids.

513 Nevertheless, the difference in preservation rates estimated in each clade $(\sim 3$ in apes

514 compared to 2.6 in cercopithecoids) should ameliorate some of the potential effect of

515 this bias on parameter estimates. The general pattern of higher net diversification in

516 cercopithecoids is also consistent with previous neontological results (Purvis et al. 1995),

517 suggesting that the paleontological estimates presented here are adequately robust to

518 these effects. This difference in lineage diversification rates between both taxa

519 demonstrates that increased morphological disparification is, overall, not correlated with

520 faster lineage diversification in haplorrhine primates. While the evolutionary

521 predisposition conferred upon apes by their relaxed integration corresponds to an increase

522 in morphological disparity, this effect does not extend to lineage diversity.

523 On the surface, the overall similarity in the environments, dietary niches, and

524 geographical ranges of hominoids and cercopithecoids suggests that cercopithecoids have

525 a selective advantage in the species-sorting dynamics between the two clades. However,

526 the long-term persistence of hominoids and their remarkable innovation in locomotor

527 function relative to cercopithecoids throughout this timespan suggests an equilibrium in 
528 their relative abundances (Van Valen 1975, Chesson 2000). Although cercopithecoids

529 experienced less dramatic innovation in postcranial morphology, they display a broader

530 range of derived digestive physiologies, including both intestinal and dental features

531 associated with food processing (Hunt 2016). Given these observations, it is possible that

532 the differential in diversification rate between cercopithecoids and hominoids stems from

533 differences in the timescales of their life histories and other factors not directly related to

534 interspecific competitive ability. If this is the case, hominoids may achieve steady

535 persistence through higher evolvability conferred by reduced integration and increased

536 developmental flexibility when faced with changing environments, while cercopithecoids

537 may do so through a combination of their higher net diversification rates and capability to

538 exploit a range of dietary resources through their digestive physiologies. Such differences

539 would be consistent with an equilibrium model of coexistence rather than a simple

540 species selection model that predicts the ultimate extinction of hominoids through

541 competitive exclusion by cercopithecoids. Nevertheless, more rigorous comparison of

542 each of these attributes in both lineages is needed to further constrain the range of

543 possible explanatory factors.

544 Constrained Markovian diffusion and entropy

545 The patterns in character disparity shown here evoke a Markovian diffusion that is

546 dampened by constraint. The tightly integrated nature of vertebrate body plans suggests

547 major constraints in their ability to fill morphospace. However, the pattern displayed by

548 apes suggests that the structure of integration may sometimes shift, allowing diffusion

549 into new areas. Morphological evolution might then be conceived as a multivariate 
550 diffusion in morphospace where movement is directionally biased due to fitness

551 differences modelled by a traditional Simpsonian adaptive landscape and certain regions

552 are rendered inaccessible by structural interactions between characters. Although this

553 diffusive model, including the feedback links between phenotype, development, and

554 environment, has been considered previously (Fisher 1986), its behavior has not been

555 well explored in this context.

556 The Monte Carlo simulations presented here provide a theoretical illustration of

557 the population-level dynamics stemming from one possible set of integration scenarios

558 and their potential effects on the evolutionary disparity/rate along a single branch in

559 Figures 3 or 4 . The results shed light on the empirical analyses by demonstrating the

560 overlapping and distinct empirical patterns that can result from varying levels of

561 developmental and functional integration in a simple closed system in the absence of

562 environmental fluctuation (Fig. 6). As is expected from quantitative genetic theory

563 (Cheverud 1984, Maynard-Smith et al. 1985), developmental and functional constraints

564 can generate similar patterns (Fig. 6a and 6b). In all these cases, the overall entropy

565 observed in the system over time remains low, with the system tending to become stuck

566 or moving away very slowly from a suboptimal location (Fig 4c and 4e) or crawling

567 slowly in concert along a gradient (Fig 6a and 6b). However, when both types of

568 constraint are released, the system displays high entropy, with each trait able to

569 independently jump between peaks (Fig. 6d). Such unconstrained movement would be

570 expected to result in higher phenotypic disparity over macroevolutionary timescales by 
571 freeing individual lineages to generate novel character combinations when exposed to

572 distinct and changing environments.

573 The patterns in increased evolutionary rate and entropy across traits displayed by

574 the less constrained simulated diffusions provide mechanistic explanations that are

575 consistent with the bursts in evolutionary rate encountered throughout hominoid

576 evolution. Although it is not possible in the empirical example to distinguish between

577 functional and developmental integration as is done in the simulations, the general pattern

578 of reduced integration that gives way to increased evolutionary rate is consistent between

579 the two. Alternation between the varying strengths of integration as explored in the

580 simulations would likely generate substantial novelty during periods of relaxed

581 integration. Episodes of reduced developmental constraint (Figs. 2 and 5a) may have

582 generated an initial burst of constructional opportunity that lead to rapid morphological

583 diversification as ape populations were exposed to highly variable environments

584 throughout the mid-Miocene. If the processes modelled in the simulations drove the

585 patterns revealed in the empirical analyses, this sequence of events would represent true

586 preadaptation in the sense that relaxed integration leads to increased mean population

587 fitness. Under the evolutionary models explored here and elsewhere (Wagner 2018),

588 stabilizing effects such as developmental canalization (Waddington 1959) or functional

589 covariance (Cheverud 1984) would then be expected to re-constrain the newly divergent

590 phenotypes after an initial burst in disparification. This dampened diffusive model was

591 explored in early work in theoretical morphology that focused on the constrained filling

592 of morphospace (Raup 1968). 
594 revealed here hint at the source of teleological concepts in human evolution. If the

595 morphological and neurological traits can be assumed to have followed a multivariate

596 Markovian diffusion that is at least qualitatively similar to the simulations in Figure 6

597 (especially Fig. 6a), the pattern in evolutionary rates along the path leading to humans

598 may paint a misleading view that such changes have followed a progressive trend leading

599 to a human-defined apex. However, the mosaic analysis here suggests that humans'

600 biological uniqueness can be attributed to a relatively small number of anatomical

601 rearrangements built over a longer diffusion into an area of developmental-morphospace

602 occupied by all apes that is characterized by increased structural opportunity. Instead of

603 treating humans as exceptional, this view suggests that the structural opportunities that

604 emerged early in ape evolution may have freed the ancestors of currently extant taxa to

605 blaze unique trajectories along a complex, multivariate adaptive landscape that would

606 have been otherwise inaccessible. Structurally, all ape species might therefore be viewed

607 as equally progressive in their evolution, with species-level differences in form and

608 function shaped by stochastic differences in separate realized evolutionary paths along a

609 shared adaptive landscape. Alternatively, separate taxa may have forged new and unique

610 adaptive landscapes following speciation events as structural differences between newly

611 isolated species drove the creation of new functional niches early in hominoid evolution.

612 The derived structure of integration and neurological development shared by chimps and

613 humans highlights the importance of contingency in human evolution-chimps' sharing

614 of much of the same underlying constructional architecture shows how this derived 
615 predisposition allowed, but did not determine, the emergence of humans' unique suite of

616 phenotypes.

617 The empirical and simulated analyses presented here evoke a picture of

618 morphological evolution that involves episodic diffusion across adaptive zones.

619 However, the empirical and theoretical analyses allow for alternating and interacting

620 roles for both ecological and constructional opportunity. This view can supplement

621 existing conceptions of adaptive radiation and key innovation by providing an alternative

622 to ecological opportunity as a lone driving factor in the disparification of form. Instead,

623 phenotypic disparity produced through diffusion into a set of abundant adaptive zones

624 can be directed and limited by the availability of structural opportunity. Nature surely

625 merges these simplified extremes when shaping patterns in biodiversity (Seilacher 1991),

626 and so further integrative study of the interplay between ecological and constructional

627 factors will be critical in developing a pluralistic understanding of the complex patterns

628 and processes that have shaped the vast diversity across the tree of life.

629

630

631

632

633

\section{References}

635 Alberch P. 1980 Ontogenesis and morphological diversification. American Zoologist 20, $636 \quad 653-667$. 
637 Andrews P. 1992 Evolution and environment in the Hominoidea. Nature 360, 641-646.

$638 \quad($ doi: $10.1038 / 360641 \mathrm{a} 0)$

639 Arnold SJ. 1992 Constraints on phenotypic evolution. American Naturalist, 140(Supp):

$640 \quad$ S85-S107.

641 Berge C. 1998 Heterochronic processes in human evolution: An ontogenetic analysis of 642 the hominid pelvis. American Journal of Physical Anthropology 105, 441-459.

643 (doi:10.1002/(sici)1096-8644(199804)105:4<441::aid-ajpa4>3.0.co;2-r)

644 Bock J. 1959 Preadaptation and multiple evolutionary pathways. Evolution 13, 194-211.

645 Boddy AM, McGowen MR, Sherwood CC, Grossman LI, Goodman M, Wildman DE.

6462012 Comparative analysis of encephalization in mammals reveals relaxed

647 constraints on anthropoid primate and cetacean brain scaling. Journal of

648 Evolutionary Biology 25, 981-994. (doi:10.1111/j.1420-9101.2012.02491.x)

649 Bouckaert R, Heled J, Kühnert D, Vaughan T, Wu C-H, Xie D, Suchard MA, Rambaut

650 A, Drummond AJ. 2014 BEAST 2: A Software Platform for Bayesian

$651 \quad$ Evolutionary Analysis. PLoS Computational Biology 10, e1003537.

$652 \quad$ (doi:10.1371/journal.pcbi.1003537)

653 Capellini I, Venditti C, Barton, RA. 2010 Phylogeny and metabolic scaling in mammals.

$654 \quad$ Ecology, 91, 2783-2793.

655 Charlesworth B, Lande R, Slatkin M. 1982 A Neo-Darwinian Commentary on

656 Macroevolution. Evolution 36, 474-498. (doi:10.1111/j.1558-

$657 \quad$ 5646.1982.tb05068.x) 
658 Chesson P. 2000 Mechanisms of maintenance of species diversity. Annual Review of

659 Ecology and Systematics, 31, 343-366.

660 Cheverud JM. 1984 Quantitative genetics and developmental constraints on evolution by 661 selection. Journal of Theoretical Biology 110, 155-171. (doi:10.1016/s0022-

662 $5193(84) 80050-8)$

663 Crouch NMA, Ricklefs RE. 2019 Speciation Rate Is Independent of the Rate of

664 Evolution of Morphological Size, Shape, and Absolute Morphological

665 Specialization in a Large Clade of Birds. The American Naturalist 193, E78-E91.

$666 \quad($ doi:10.1086/701630)

667 Donoghue MJ, Ree RH. 2000 Homoplasy and Developmental Constraint: A Model and

668 an Example from Plants. American Zoologist 40, 759-769.

669 (doi:10.1093/icb/40.5.759)

670 Donoghue MJ. 2005 Key innovations, convergence, and success: macroevolutionary

671 lessons from plant phylogeny. Paleobiology 31, 77-93. (doi: 10.1666/0094-

672 8373(2005)031[0077:KICASM]2.0.CO;2)

673 Felice RN, Goswami A. 2017 Developmental origins of mosaic evolution in the avian 674 cranium. Proceedings of the National Academy of Sciences 115, 555-560.

$675 \quad$ (doi:10.1073/pnas.1716437115)

676 Felsenstein J. 1985 Phylogenies and the comparative method. The American Naturalist, $677 \quad 125,1-15$.

678 Fisher, DC. 1986 Progress in organismal design. Pp. 99-117 in: Patterns and Processes in 679 the History of Life. Raup DM and Jablonski D, eds. Springer, Berlin, Heidelberg. 
680 Goswami A, Binder WJ, Meachen J, O’Keefe FR. 2015 The fossil record of phenotypic

681 integration and modularity: A deep-time perspective on developmental and

682 evolutionary dynamics. Proceedings of the National Academy of Sciences 112,

683 4891-4896. (doi:10.1073/pnas.1403667112)

684 Goswami A, Smaers JB, Soligo C, Polly PD. 2014 The macroevolutionary consequences

685 of phenotypic integration: from development to deep time. Philosophical

686 Transactions of the Royal Society B: Biological Sciences 369, 20130254-

687 20130254. (doi:10.1098/rstb.2013.0254)

688 Gould SJ, Vrba ES. 1982 Exaptation — a Missing Term in the Science of Form.

689 Paleobiology 8, 4-15. (doi:10.1017/s0094837300004310)

690 Gould SJ and Lewontin RC. 1979 The spandrels of San Marco and the Panglossian

691 paradigm: a critique of the adaptationist programme. Proceedings of the Royal

692 Society of London. Series B. Biological Sciences 205, 581-598.

693 (doi:10.1098/rspb.1979.0086)

694 Gould, SJ. 1977 Ontogeny and Phylogeny. Harvard University Press, Cambridge, USA.

695 Harmon LJ. 2003 Tempo and Mode of Evolutionary Radiation in Iguanian Lizards.

696 Science 301, 961-964. (doi:10.1126/science.1084786)

697 Holloway RL. 1973 Endocranial volumes of early African hominids, and the role of the 698 brain in human mosaic evolution. Journal of Human Evolution 2, 449-459.

699 (doi:10.1016/0047-2484(73)90123-1)

700 Hunt KD. 2016 Why are there apes? Evidence for the co-evolution of ape and monkey 701 ecomorphology. Journal of Anatomy 228, 630-685. (doi:10.1111/joa.12454) 
702 Lewton KL. 2010 Locomotor function and the evolution of the primate pelvis. (Doctoral 703 dissertation, Arizona State University).

704 Losos JB. 2010 Adaptive Radiation, Ecological Opportunity, and Evolutionary

705 Determinism. The American Naturalist 175, 623-639. (doi:10.1086/652433)

706 Mahler DL, Ingram T, Revell LJ, Losos JB. 2013 Exceptional Convergence on the

707 Macroevolutionary Landscape in Island Lizard Radiations. Science 341, 292-295.

708 (doi:10.1126/science.1232392)

McHenry H. 1975 Fossils and the mosaic nature of human evolution. Science 190, 425431. (doi:10.1126/science.809842)

711 Michel LA et al. 2014 Remnants of an ancient forest provide ecological context for Early Miocene fossil apes. Nature Communications 5. (doi:10.1038/ncomms4236)

713 Rice, SH. 2002. The role of heterochrony in primate brain evolution. In: Human evolution through developmental change. N. Minugh-Purvis \& K.J. McNamara,

716 Seilacher A. 1991 Self-Organizing Mechanisms in Morphogenesis and Evolution. In

717 Constructional Morphology and Evolution, pp. 251-271. Springer Berlin

718 Heidelberg. (doi:10.1007/978-3-642-76156-0_17)

719 Silvestro D, Salamin N, Schnitzler J. 2014 PyRate: a new program to estimate speciation 720 and extinction rates from incomplete fossil data. Methods in Ecology and $721 \quad$ Evolution 5, 1126-1131.

722 Smith JM, Burian R, Kauffman S, Alberch P, Campbell J, Goodwin B, Lande R, Raup D, 723 Wolpert L. 1985 Developmental Constraints and Evolution: A Perspective from 
the Mountain Lake Conference on Development and Evolution. The Quarterly Review of Biology 60, 265-287. (doi:10.1086/414425)

726 Smith SA, Brown JW, Yang Y, Bruenn R, Drummond CP, Brockington SF, Walker JF, Last N, Douglas N, Moore MJ. 2018 Disparity, Diversity, and Duplications in the Caryophyllales. New Phytologist 217, 836-854.

Olson ME. 2012 The developmental renaissance in adaptationism. Trends in Ecology and Evolution 27, 278-287. (doi:10.1016/j.tree.2011.12.005)

731 Olson EC and Miller RL. 1958. Morphological Integration. University of Chicago Press.

732 Parins-Fukuchi C. 2018 Bayesian placement of fossils on phylogenies using quantitative morphometric data. Evolution 72, 1801-1814. (doi:10.1111/evo.13516)

734 Parins-Fukuchi C. In press. Detecting mosaic patterns in macroevolutionary disparity. American Naturalist.

Purvis A, Nee S, Harvey PH. 1995 Macroevolutionary inferences from primate Sciences 260, 329-333.

739 Rabosky DL, Santini F, Eastman J, Smith SA, Sidlauskas B, Chang J, Alfaro ME. 2013 Rates of speciation and morphological evolution are correlated across the largest vertebrate radiation. Nature Communications 4. (doi:10.1038/ncomms2958)

742 Rabosky DL. 2014 Automatic Detection of Key Innovations, Rate Shifts, and DiversityDependence on Phylogenetic Trees. PLoS One 9, e89543. 
745 Rabosky DL, Grundler M, Anderson C, Title P, Shi JJ, Brown JW, Huang H, Larson JG.

2014 BAMMtools: an R package for the analysis of evolutionary dynamics on

747 phylogenetic trees. Methods in Ecology and Evolution 5, 701-707.

$748 \quad$ (doi:10.1111/2041-210x.12199)

749 Rainey PB, Travisano M. 1998 Adaptive radiation in a heterogeneous environment.

$750 \quad$ Nature 394, 69-72. (doi:10.1038/27900)

751 Raup DM. 1968 Theoretical morphology of echinoid growth. Journal of Paleontology 42, $752 \quad 50-63$.

753 Ruff CB. 1994 Morphological adaptation to climate in modern and fossil hominids.

754 American Journal of Physical Anthropology 37, 65-107.

$755 \quad$ (doi:10.1002/ajpa.1330370605)

756 Simpson GG. 1944 Tempo and Mode in Evolution. Columbia University Press. New $757 \quad$ York.

758 Slater GJ, Friscia AR. 2019 Hierarchy in adaptive radiation: A case study using the 759 Carnivora (Mammalia). Evolution 73, 524-539. (doi:10.1111/evo.13689)

760 Slater GJ. 2015 Iterative adaptive radiations of fossil canids show no evidence for 761 diversity-dependent trait evolution. Proceedings of the National Academy of 762 Sciences 112, 4897-4902. (doi:10.1073/pnas.1403666111)

763 Sukumaran J, Holder MT. 2010 DendroPy: a Python library for phylogenetic computing. 764 Bioinformatics 26, 1569-1571. (doi:10.1093/bioinformatics/btq228)

765 Stebbins GL. 1983 Mosaic evolution: An integrating principle for the modern synthesis.

766 Experientia 39, 823-834. (doi:10.1007/bf01990398) 
767 Stebbins GL. 1984 Mosaic evolution, mosaic selection and angiosperm phylogeny.

768 Botanical Journal of the Linnean Society 88, 149-164. (doi:10.1111/j.1095-

769 8339.1984.tb01568.x)

770 Van Valen L. 1975 Group selection, sex, and fossils. Evolution, 29, 87-94.

771 Vos RA. 2006 Inferring large phylogenies: the big tree problem (Doctoral dissertation, $772 \quad$ Simon Fraser University)

773 Waddington CH. 1959 Canalization of Development and Genetic Assimilation of

774 Acquired Characters. Nature 183, 1654-1655. (doi:10.1038/1831654a0)

775 Wagner CE, Harmon LJ, Seehausen O. 2012 Ecological opportunity and sexual selection 776 together predict adaptive radiation. Nature 487, 366-369.

777 (doi:10.1038/nature11144)

778 Wagner GP, Pavlicev M, Cheverud JM. 2007 The road to modularity. Nature Reviews 779 Genetics 8, 921-931.

780 Wagner GP, Amemiya C, Ruddle F. 2003 Hox cluster duplications and the opportunity 781

782 for evolutionary novelties. Proceedings of the National Academy of Sciences 100, 14603-14606. (doi:10.1073/pnas.2536656100)

783 Wagner GP. 1988 The influence of variation and of developmental constraints on the rate 784 of multivariate phenotypic evolution. Journal of Evolutionary Biology 1, 45-66.

785 (doi:10.1046/j.1420-9101.1988.1010045.x)

786 Wagner PJ. 2018 Early bursts of disparity and the reorganization of character integration.

787 Proceedings of the Royal Society B: Biological Sciences 285, 20181604.

788 (doi:10.1098/rspb.2018.1604) 
789 Worthington S. 2012 New approaches to late Miocene hominoid systematics: Ranking

790 morphological characters by phylogenetic signal (Doctoral dissertation, New

$791 \quad$ York University).

792 Wright, S. 1932 The roles of mutation, inbreeding, crossbreeding and selection in

793 evolution. Proc. 6th Int. Cong. Genet. 1: 356-366.

794 Yoder JB, et al. 2010 Ecological opportunity and the origin of adaptive radiations.

795 Journal of Evolutionary Biology 23, 1581-1596. (doi:10.1111/j.1420-

$796 \quad 9101.2010 .02029 . x)$

797 Young NM, Wagner GP, Hallgrímsson B. 2010 Development and the evolvability of

798 human limbs. Proceedings of the National Academy of Sciences 107, 3400-3405.

$799 \quad$ (doi:10.1073/pnas.0911856107)

800

801

802

803

804

805

806

807

808

809

810 Tables 
bioRxiv preprint doi: https://doi.org/10.1101/622134; this version posted October 11,2019 . The copyright holder for this preprint (which was not certified by peer review) is the author/funder, who has granted bioRxiv a license to display the preprint in perpetuity. It is made available under aCC-BY-NC-ND 4.0 International license.

\section{1}

\begin{tabular}{|l|l|l|l|l|}
\hline Clade & Origination & Extinction & Net diversification & Preservation \\
\hline Hominoidea & 0.395 & 0.338 & 0.057 & 3.121 \\
\hline Cercopithecoidea & 0.251 & 0.128 & 0.123 & 2.551 \\
\hline
\end{tabular}

812 Table 1. Lineage diversification and preservation rate parameters estimated in apes

813 (Hominoidea) and Old World monkeys (Cercopithecoids). Parameters were summarized

814 as the mean values from four million Markov-Chain Monte Carlo generations after

815 discarding $20 \%$ as burn-in.

816

817

818

819

820

821

822

823

824

825

826

827

828

829 Figures 


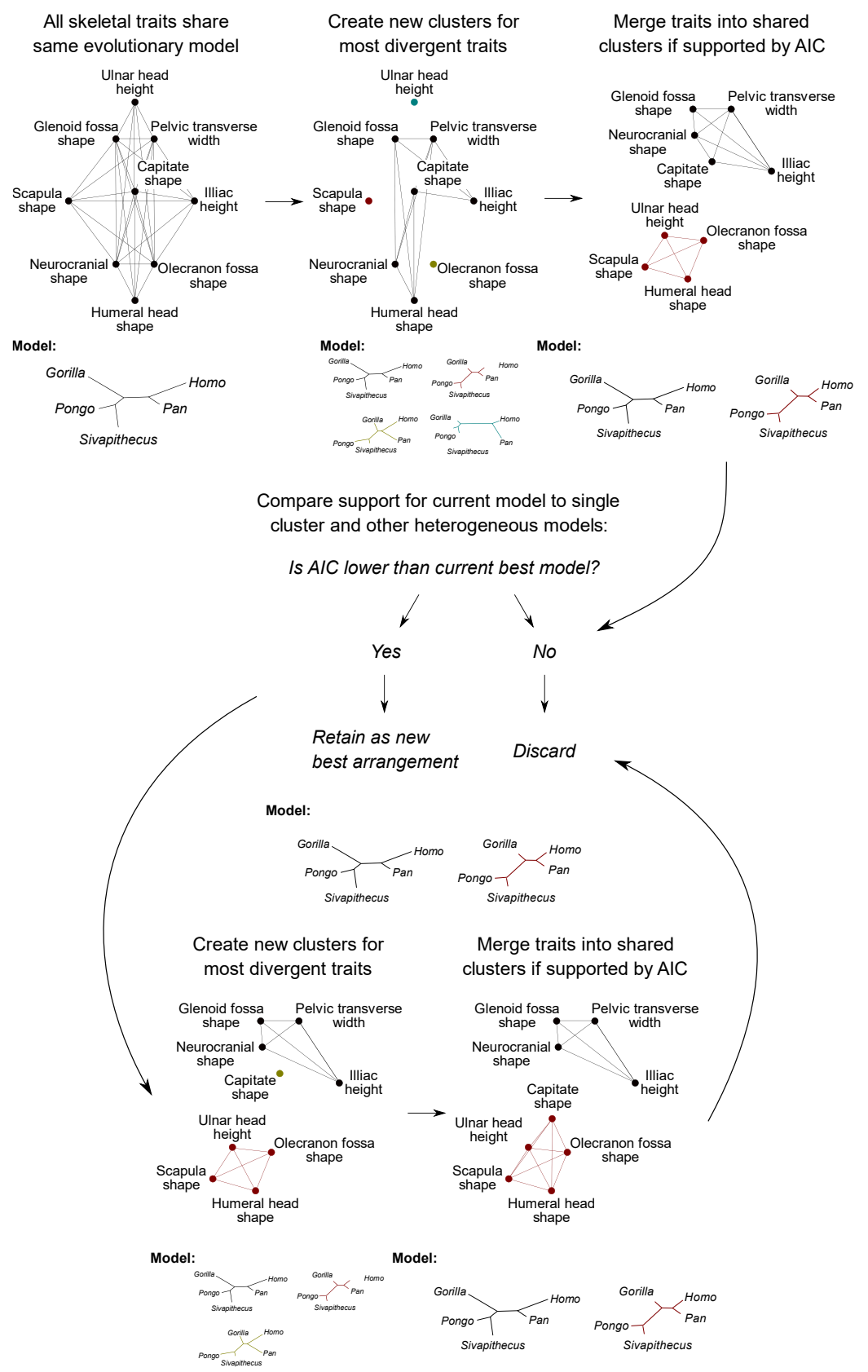

831 Figure 1. The mosaic inference procedure starts by modelling all characters under a

832 unified phylogenetic model, in which branch lengths represent morphological disparity.

833 The algorithm then splits traits with divergent patterns in disparity into their own suites

834 and then greedily merges these suite fragments until the AIC score ceases to improve.

835 The splitting and merging steps are then alternated until the AIC scores of the final 39 
836 merged models cease to improve. The best clustering achieved from this procedure

837 represents the best-supported heterogeneous model of character integration.

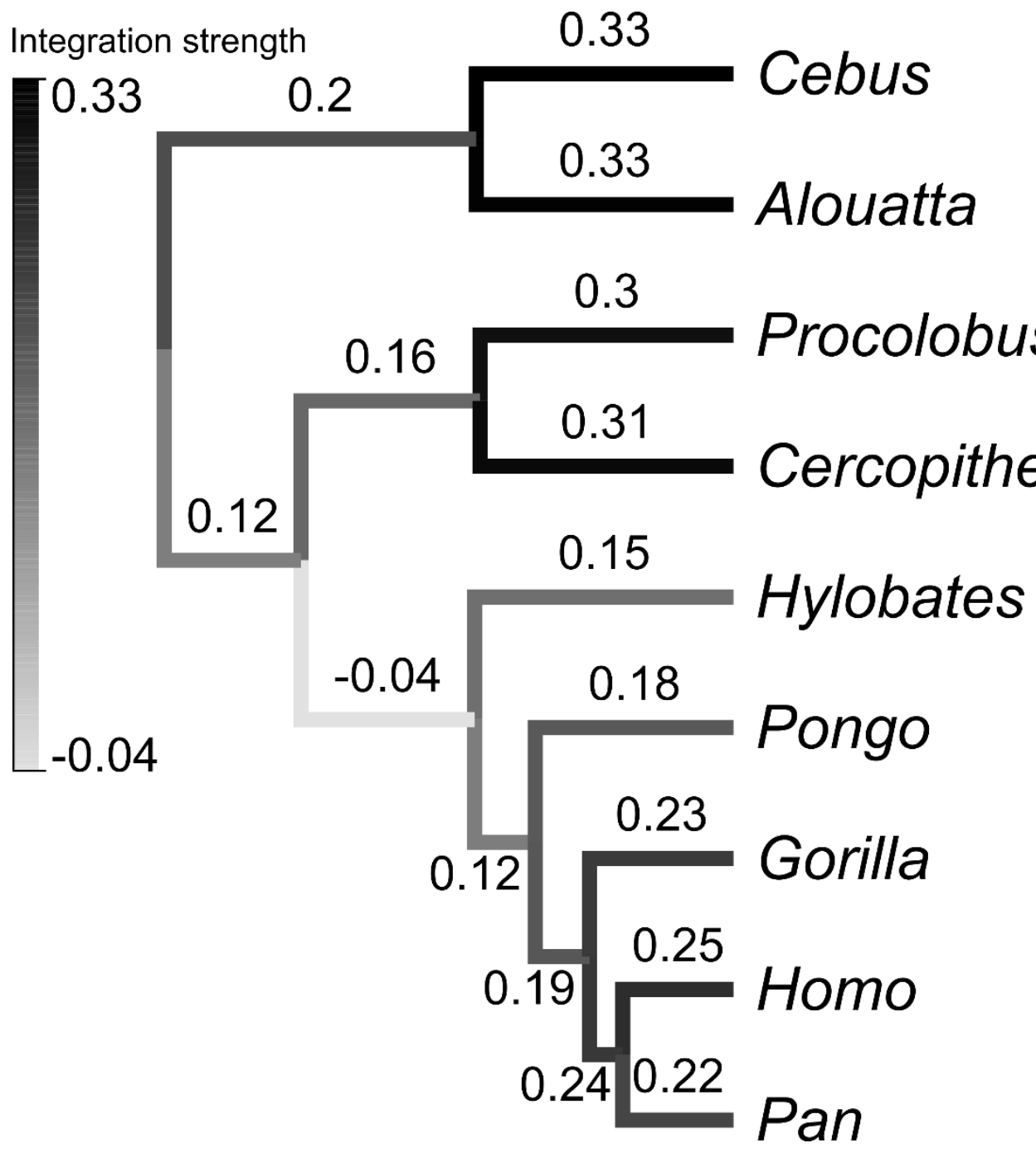

839 Figure 2. Strength of integration across haplorrhine evolution. Branch labels correspond

840 to the strength and polarity of correlation in the reconstructed changes along each branch.

841 Higher correlations in either direction (closer to either 1 or -1 ) indicate stronger

842 evolutionary integration, while lower correlations (closer to 0) indicate weaker

843 integration. 

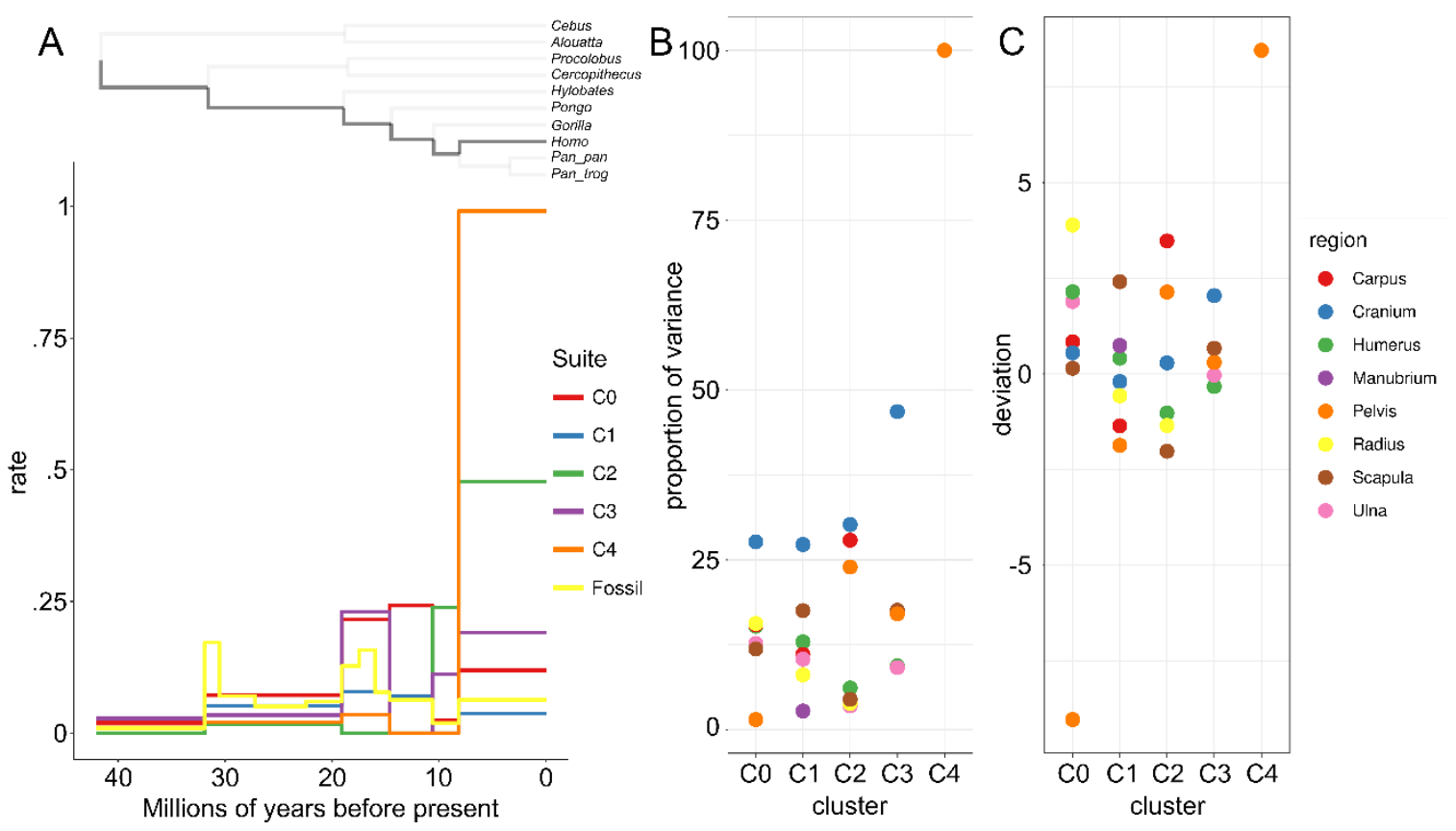

845 Figure 3. A) Mosaic evolutionary rates calculated while walking back from Homo to the

846 most recent common ancestor of New World monkeys, Old World Monkeys, and apes.

847 B) Variance contributed to each suite by each skeletal region, estimated from a principal

848 component analysis. C) Skeletal regions over-represented in each mosaic evolutionary

849 suite. Values above zero correspond to regions that occupy a higher proportion of their

850 clusters than expected.

851 


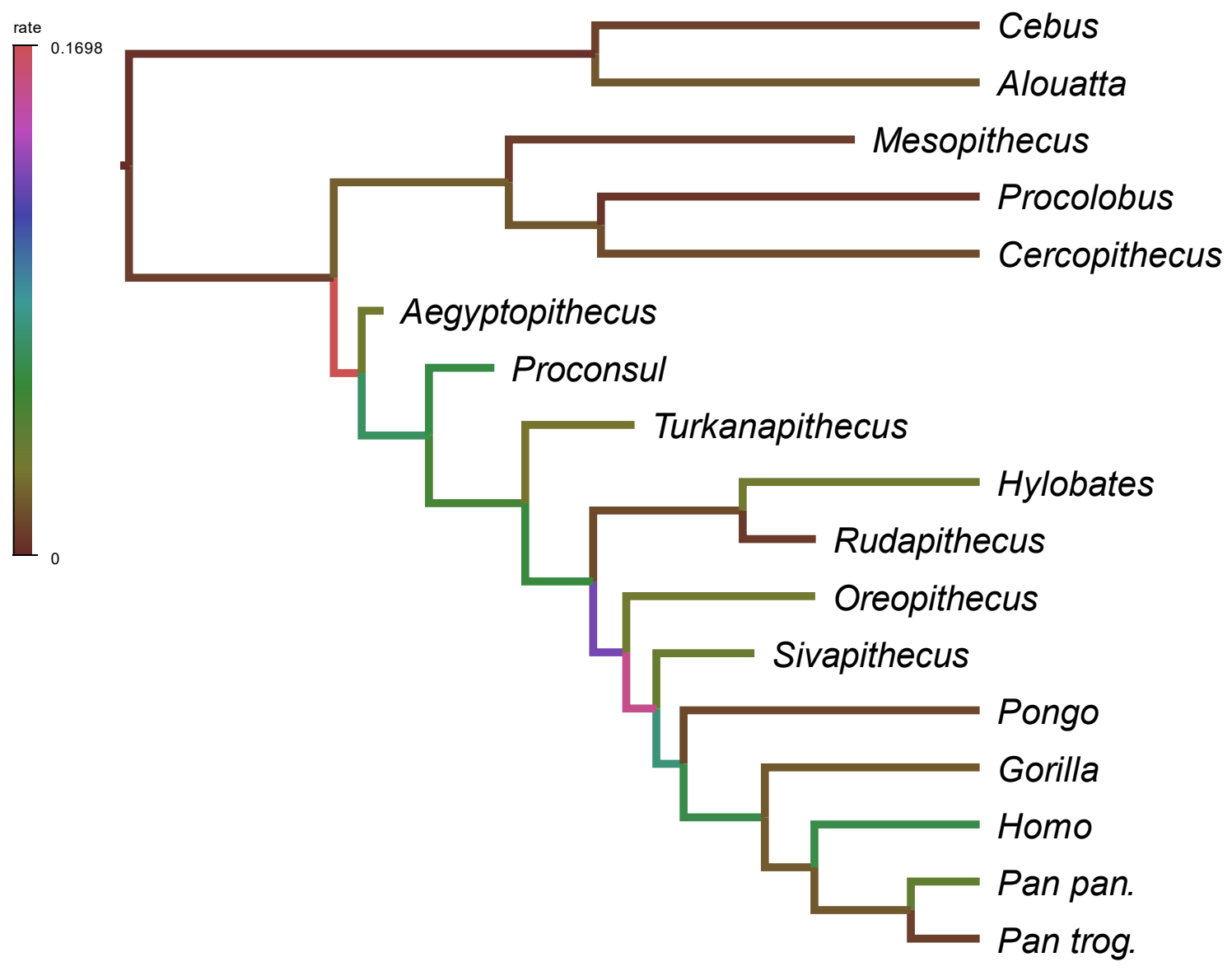

853 Figure 4. Branch colors correspond to rates of morphological evolution across all

854 characters along individual branches. Phylogenetic positions of fossil genera reflect

855 placements on extant reference tree.

856

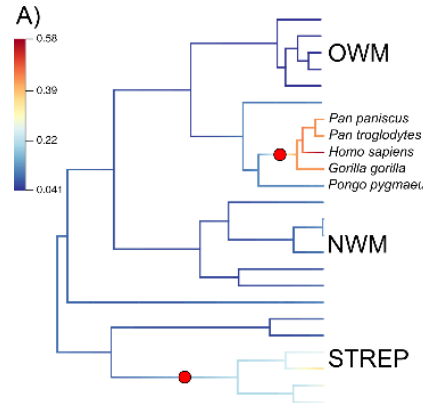

857

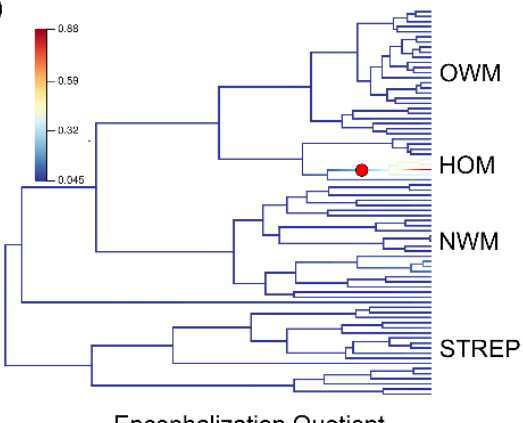

Encephalization Quotient

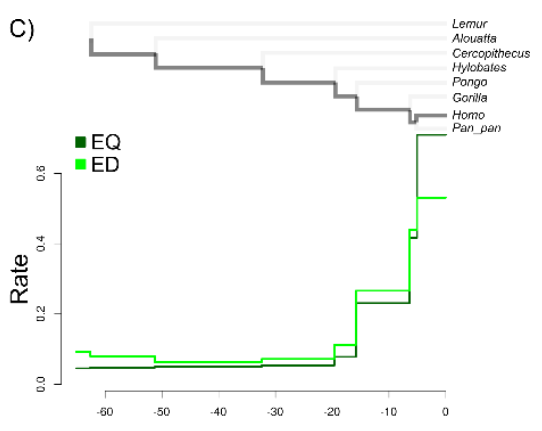

Myr before present 
bioRxiv preprint doi: https://doi.org/10.1101/622134; this version posted October 11,2019 . The copyright holder for this preprint (which was not certified by peer review) is the author/funder, who has granted bioRxiv a license to display the preprint in perpetuity. It is made available under aCC-BY-NC-ND 4.0 International license.

858 Figure 5. Rate shifts in A) encephalization development, and B) encephalization quotient.

859 C) Branch-specific evolutionary rates when tracing the lineage from Homo to the root.
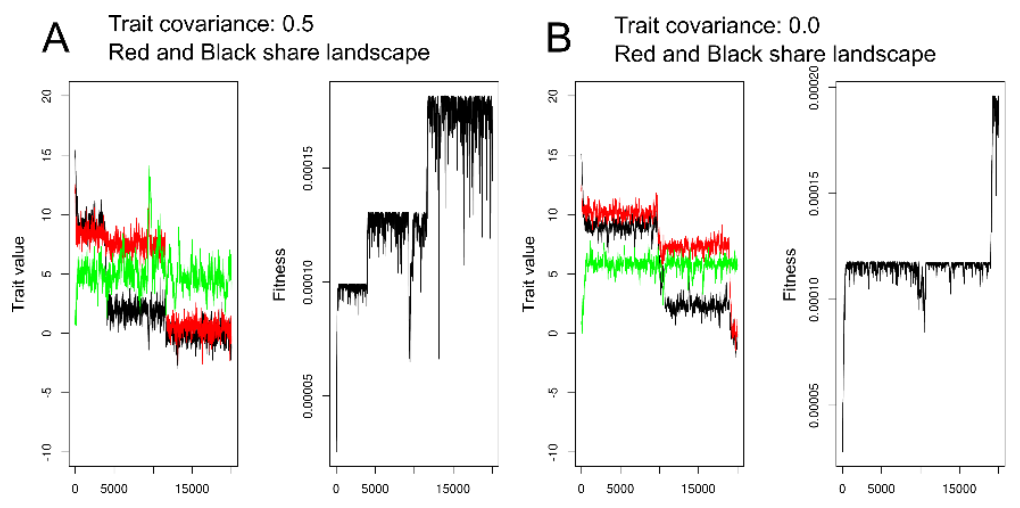

C Trait covariance: 0.0
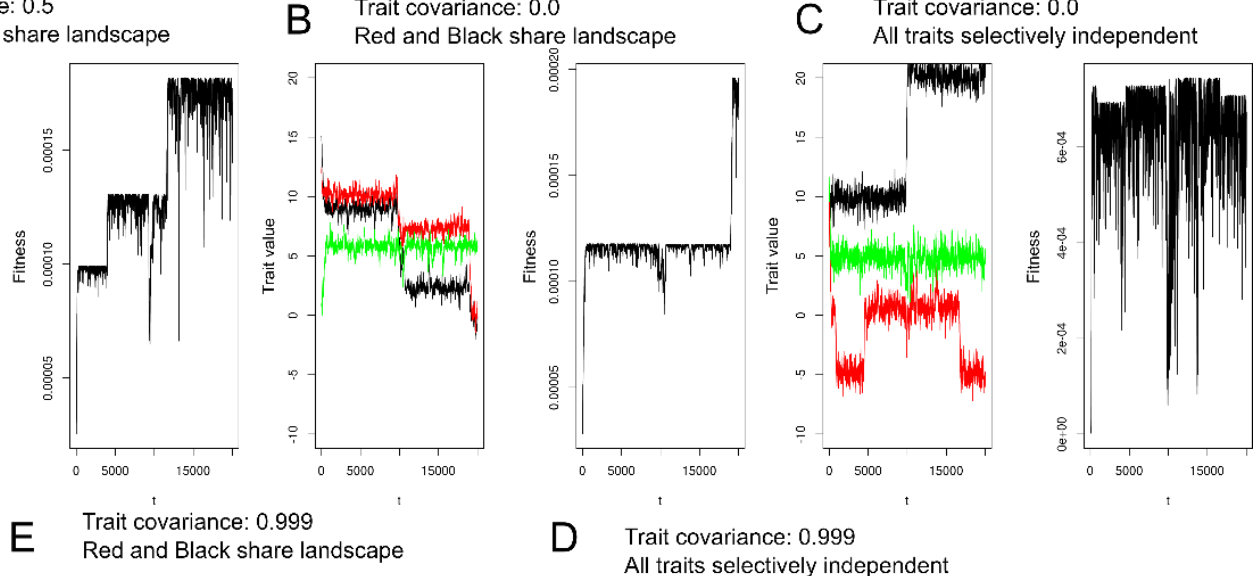

860
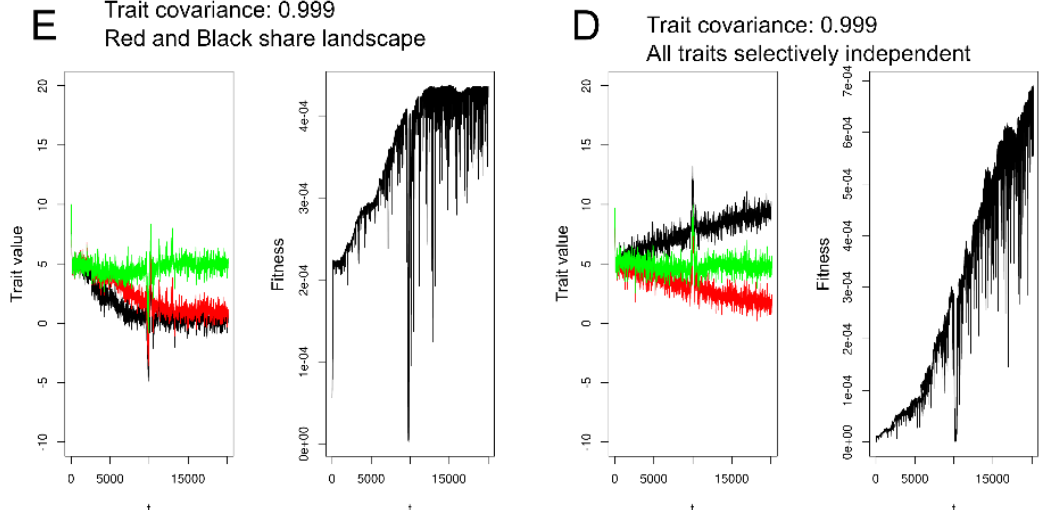

861 Figure 6. Monte Carlo simulations of integrated and atomized systems of three

862 continuous traits. Each box is a simulated diffusion between three traits under A)

863 moderate developmental linkage and shared selection, B) no developmental linkage and

864 shared selection, C) no developmental linkage and selective independence, D) high

865 developmental linkage and selective independence, and E) high developmental linkage

866 and shared selection.

867

868

869

870 


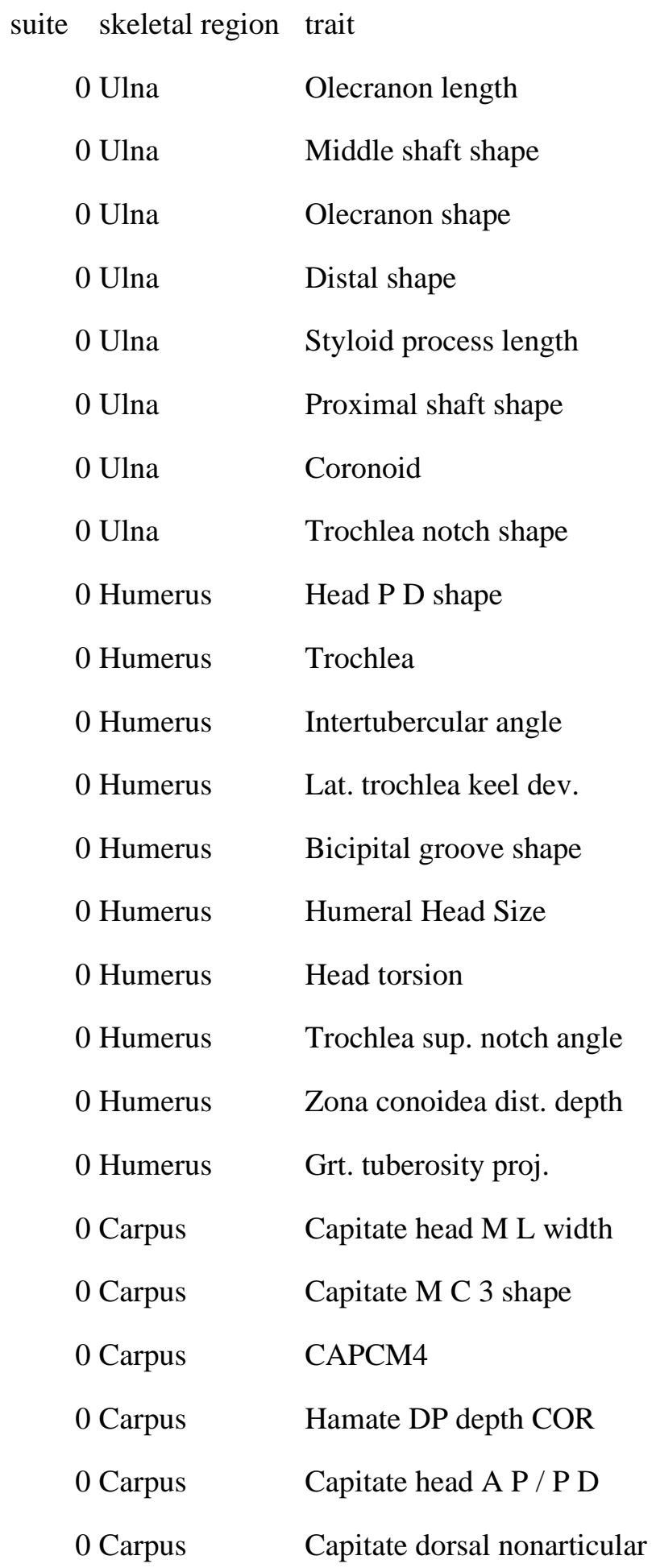




\begin{tabular}{ll} 
0 Carpus & Capitate M C 3 depth \\
0 Carpus & Lunate dist. \\
0 Carpus & Hamate triquetral facet angle \\
0 Carpus & Capitate M C 2 facets \\
0 Cranium & Grt. palatine foramen position \\
0 Cranium & Zygomatic root origin elevation \\
0 Cranium & Basicranial length \\
0 Cranium & Piriform aperture shape \\
0 Cranium & Postglenoid projection \\
0 Cranium & Basicranium vs. palate \\
0 Cranium & Bregma position \\
0 Cranium & Lacrimal fossa \& orbital floor \\
0 Cranium & Facial projection \\
0 Cranium & Zygomax. tubercle A P width \\
0 Cranium & Basicranium vs. nuchal \\
0 Cranium & Nasoalveolar clivus length \\
0 Cranium & Head M L shape \\
0 Cranium & Zygomatic \\
\hline 0 Cranium & Face height vs. depth \\
\hline O Cranium & Piriform max. M L elevation \\
\hline Neck shape
\end{tabular}




\begin{tabular}{|c|c|}
\hline 0 Radius & Distal facets area \\
\hline 0 Radius & Total length \\
\hline 0 Radius & Radial neck length \\
\hline 0 Radius & Shaft curvature M L \\
\hline 0 Radius & Head articular surface P D \\
\hline 0 Scapula & Trapezius insert angle \\
\hline 0 Scapula & Coraco \\
\hline 0 Scapula & Inf. glenoid cor \\
\hline 0 Scapula & Acromian size \\
\hline 0 Scapula & Inferior angle \\
\hline 0 Scapula & Sup. notch shape \\
\hline 0 Scapula & Spinatus ratio \\
\hline 0 Scapula & Glenoid vent. bar angle \\
\hline 0 Pelvis & pubic symphysis length \\
\hline 1 Ulna & Ulna head height \\
\hline 1 Ulna & Semilunar keel angle \\
\hline 1 Ulna & Radial notch shape \\
\hline 1 Ulna & Distal shaft shape \\
\hline 1 Humerus & Dist. articular shape \\
\hline 1 Humerus & Trochlea dist. waisting \\
\hline 1 Humerus & Med. trochlea keel dev. \\
\hline 1 Humerus & Olecranon fossa depth \\
\hline 1 Humerus & Olecranon fossa shape \\
\hline 1 Carpus & Capitate P D length \\
\hline 1 Carpus & Hamate M C 4 \\
\hline 1 Carpus & Lunate radial facet $\mathrm{ML}$ \\
\hline 1 Carpus & Lunate triquetral facet shape \\
\hline
\end{tabular}




\begin{tabular}{|c|c|}
\hline 1 Cranium & Piriform base M L width \\
\hline 1 Cranium & M1 shape \\
\hline 1 Cranium & Lsr. palatine foramen \\
\hline 1 Cranium & Molar crown areas \\
\hline 1 Cranium & Entoglenoid width \\
\hline 1 Cranium & Entoglenoid projection \\
\hline 1 Cranium & Nasal bone M L width \\
\hline 1 Cranium & Orbital margin $\mathrm{M} \mathrm{L}$ width \\
\hline 1 Cranium & Orbit area \\
\hline 1 Cranium & Interorbital width \\
\hline 1 Radius & Distal surface shape \\
\hline 1 Radius & Styloid process length \\
\hline 1 Radius & Radial Head Shape \\
\hline 1 Manubrium & Manubrium shape \\
\hline 1 Scapula & Scapula Body shape \\
\hline 1 Scapula & Trapezius med. insert \\
\hline 1 Scapula & Superior angle \\
\hline 1 Scapula & Subscapular depth \\
\hline 1 Scapula & Vertebral border length \\
\hline 1 Scapula & Glenoid fossa shape \\
\hline 1 Scapula & Coracoid length \\
\hline 1 Pelvis & illium length \\
\hline 1 Pelvis & bi \\
\hline 1 Pelvis & anteroposterior pelvic diameter \\
\hline 1 Pelvis & pelvic length \\
\hline 2 Humerus & Epicondyles projection \\
\hline 2 Humerus & Olecranon perforation \\
\hline
\end{tabular}


2 Ulna

2 Carpus

2 Carpus

2 Carpus

2 Carpus

2 Carpus

2 Carpus

2 Carpus

2 Cranium

2 Cranium

2 Cranium

2 Cranium

2 Cranium

2 Cranium

2 Cranium

2 Radius

2 Scapula

2 Pelvis

2 Pelvis

2 Pelvis

2 Pelvis

2 Pelvis

2 Pelvis

3 Scapula

3 Scapula

3 Pelvis

3 Pelvis
Shaft curvature A P

Capitate M C 3 waisting

Capitate waisting

Lunate PD Length

Hamate hamulus P D proj.

Capitate M C 2 margin

Capitate M C 2 orientation

Lunate dist. facet shape

Orbit shape

Pterion post. config

Palate shape

Lacrimal fossa visibility

Palatine width

Postorbital constriction

Palate depth

Head depth P D

Glenoid fossa depth

illium width

ischium length

AIIS to hip joint

inferior pubic ramus length

acetabulum diameter

superior pubic ramus length

Spinoglenoid notch depth

Infraglenoid tuberosity M L

lower illiac height

inferior pubic ramus cross 


$\begin{array}{ll}\text { 3 Ulna } & \text { Radial notch depth } \\
\text { 3 Cranium } & \text { Temporal fossa width } \\
\text { 3 Cranium } & \text { Face vs. nasal height } \\
\text { 3 Cranium } & \text { Face height vs. width } \\
\text { 3 Cranium } & \text { Palate width } \\
\text { 3 Cranium } & \text { Entoglenoid lat. pterygoid } \\
\text { 3 Humerus } & \text { Medial trochlea keel \& medial epicon } \\
4 \text { Pelvis } & \text { ischium cross } \\
4 \text { Pelvis } & \text { superior pubic ramus cross } \\
4 \text { Pelvis } & \text { upper illiac height } \\
4 \text { Pelvis } & \text { lower ilium cross } \\
4 \text { Pelvis } & \text { ischium dorsal projection } \\
4 \text { Pelvis } & \text { pubic symphysis } \\
4 \text { Pelvis } & \text { auricular surface area } \\
4 \text { Pelvis } & \text { biacetabular diameter } \\
4 \text { Pelvis } & \text { maximum transverse diameter } \\
4 \text { Pelvis } & \text { sacrum width }\end{array}$

873

874 Table S1: List of traits and their skeletal regions that comprise each of the mosaic suites detected 875 from the skeletal data.

876 